\title{
The CEP and Geophysical Interpretation of Modern Earth Rotation Observations
}

\author{
Aleksander Brzeziński \\ Space Research Centre, Polish Academy of Sciences, Bartycka 18A, \\ 00-716 Warsaw, Poland, e-mail: alek@cbk.waw.pl
}

\begin{abstract}
The definition of the Celestial Ephemeris Pole (CEP) which is the pole of reference for precession and nutation, should be revised taking into account recent advances in observation and theory. This paper reviews the current realization of the CEP and discusses possible extensions of both the conceptual definition and the realization of the CEP. Attention is focused on the corresponding connections between the Earth orientation parameters describing rotational variations and the related excitation parameters expressing dynamics of the geophysical fluids.
\end{abstract}

\section{Introduction}

The coordinate transformation between the Terrestrial Reference System (TRS) and the Celestial Reference System (CRS) makes use of an intermediate pole of reference, the Celestial Ephemeris Pole (CEP). The CEP was defined as a pole of reference for precession and nutation in the report presenting the International Astronomical Union (IAU) 1980 Theory of Nutation (Seidelmann, 1982); see also (Capitaine et al., 1985) and (Capitaine, 1986) for supplementary clarifications. Recently it became clear that the CEP should be redefined taking into account advances in modeling and monitoring Earth rotation (Capitaine and Brzeziński, 1998). Particular points which have to be taken into account are the following.

- Space geodetic techniques, Very Long Baseline Interferometry (VLBI) and Global Positioning System (GPS), are able to monitor changes in Earth orientation on a near-continuous basis, with a sampling interval 1 or 2 hours, and with an angular resolution of the order of 0.1 mas (milliarcsecond).

- There are diurnal and subdiurnal terms both in polar motion and nutation, which are predicted by theory and in some cases also verified by observations. This includes prograde diurnal and semidiurnal terms in nutation with amplitudes of the order of $10 \mu$ as (microarcseconds), and diurnal/semidiurnal terms in polar motion driven by the oceanic tides, with amplitudes up to several hundreds of $\mu$ as.

- There has also been significant improvement in modeling and monitoring global dynamics of the geophysical fluids, including subdiurnal estimates of the atmospheric/oceanic angular momentum. 
The purpose of this paper is to review the current realization of the CEP (sections 2 and 3 ) and consider possible extensions which take into account diurnal and subdiurnal variations in Earth rotation (section 4). Our presentation is based on the matrix transformation between the TRS and the CRS in the form proposed by Brzeziński and Capitaine (1993; 1995). The corresponding model of polar motion is extended by following the concept of Mathews (1998). We consider in detail how this extended model can be used for geophysical interpretation of the modern polar motion observations.

\section{Current realization of the CEP}

Current practice is to describe Earth rotation by the conventional IAU precession/nutation model and the following five Earth orientation parameters (EOP) which are routinely estimated from observations

- $x_{p}, y_{p}$ - the terrestrial coordinates of the CEP;

- $\delta \psi, \delta \varepsilon$ - the spatial offsets in longitude and obliquity of the CEP with respect to its position predicted by the precession/nutation model;

- UT1-UTC - variations of the universal time expressing the unpredictable part of the sidereal rotation angle around the CEP axis.

These parameters can be used in the coordinate transformation between the CRS and the TRS at the date $t$ of observation. Different forms of such transformation are described in detail in Chapter 5 of the IERS Conventions (McCarthy, 1996). Our preferable expression is that proposed by Brzeziński and Capitaine (1993; 1995)

$$
[\mathrm{TRS}]=R_{2}\left(-x_{p}\right) R_{1}\left(-y_{p}\right) R_{3}(\phi) R_{1}\left(-Y^{\prime}\right) R_{2}\left(X^{\prime}\right)[\mathrm{CRS}]^{\prime},
$$

in which $R_{l}(\alpha)$ stands for the rotation around the $l$ th axis by the angle $\alpha, \mathrm{CRS}^{\prime}$ is the celestial system after applying the conventional precession/nutation model, $X^{\prime}, Y^{\prime}$ are the incremental celestial coordinates of the CEP (referred to the pole of $\mathrm{CRS}^{\prime}$ ) and $\phi=\phi_{0}+\Omega\left(t-t_{0}\right)+\delta \phi$ denotes the angle of diurnal sidereal rotation around the CEP axis with the average angular speed $\Omega$ and the residual part $\delta \phi$ including the unpredictable variations UT1-UTC.

Let us note that

1. The incremental celestial coordinates of the CEP are related to the currently reported quantities, the EOPs, by the expressions $X^{\prime}=\delta \psi \sin \varepsilon_{0}$, $Y^{\prime}=\delta \varepsilon$, in which $\varepsilon_{0}$ is the mean obliquity at the reference epoch $t_{0}$.

2. The angle $\delta \phi$ including UT1 variations depends on the applied conventions (classical procedure, using the Conventional Ephemeris Origin, etc.), but the differences are negligible when considering only the direction of the CEP.

3. From observations it follows that $x_{p}, y_{p}=O\left(10^{-6}\right), X^{\prime}, Y^{\prime}=O\left(10^{-8}\right)$. Therefore transformation (1) involves only small rotations about the equatorial axes, which are very convenient to handle in mathematical derivations. 
From eq. (1) it can be deduced that the unit vector $\vec{e}_{C}$ along the CEP axis has the following first representations:

$$
\left(x_{p},-y_{p}, 1\right)^{T} \text { in the TRS, }\left(X^{\prime}, Y^{\prime}, 1\right)^{T} \text { in the CRS'. }
$$

The minus sign in the $y$-coordinate is due to the opposite conventions for the reported polar motion components and for the direction of the TRS axes. Let us further denote by $\vec{e}_{z}, \vec{e}_{Z^{\prime}}$ the unit vectors along the polar axes of the TRS and $\mathrm{CRS}^{\prime}$, respectively. The difference $\vec{e}_{C}-\vec{e}_{z}$ which is referred to as polar motion, is obtained as $\left(x_{p},-y_{p}, 0\right)^{T}$ in the TRS. Similarly, the difference $\vec{e}_{C}-\vec{e}_{Z^{\prime}}$, expressing the celestial perturbation of the CEP, usually referred to as nutation, has the following expression in the CRS $:\left(X^{\prime}, Y^{\prime}, 0\right)^{T}$. The third component of these differences is zero in the first order approximation, hence an equivalent and compact representation of polar motion and nutation is obtained after projecting them onto the polar planes (terrestrial and celestial, respectively, which are almost parallel but differ by the approximately uniform rotation with diurnal period) and using complex variables

$$
p=x_{p}-i y_{p}, \quad P=X^{\prime}+i Y^{\prime},
$$

where $i=\sqrt{-1}$.

\section{Basic kinematical relationships}

\subsection{Polar motion versus nutation}

The coordinate transformation (1) is described by 5 parameters, the EOP's, while from the elementary geometrical considerations it is known that only 3 parameters are necessary, hence there are 2 redundant degrees of freedom. There is full degeneracy between polar motion and nutation parameters: any (small) change $\Delta P$ of the celestial coordinates can be compensated by the change $\Delta p=\Delta P e^{-i \phi}$ of the terrestrial coordinates; see (Brzeziński and Capitaine, 1993) for derivations and extensive discussion. In the extreme case, the whole perturbation of the pole can be expressed in the terrestrial frame

$$
[\mathrm{TRS}]=R_{2}\left(-x_{p}-x_{p}^{\prime}\right) R_{1}\left(-y_{p}-y_{p}^{\prime}\right) R_{3}(\tilde{\phi})[\mathrm{CRS}]^{\prime},
$$

where

$$
x_{p}^{\prime}=-X^{\prime} \cos \phi-Y^{\prime} \sin \phi, \quad y_{p}^{\prime}=-X^{\prime} \sin \phi+Y^{\prime} \cos \phi,
$$

or, using complex variables,

$$
p^{\prime}=-P e^{-i \phi},
$$

where $p^{\prime}=x_{p}^{\prime}-i y_{p}^{\prime}$ and the rotation angle $\tilde{\phi}$ can differ slightly from that used in eq. (1) due to the change of the rotation axis. In equations (3) and (4) the sidereal rotation angle can be replaced by its linear part $\phi=\phi_{0}+\Omega\left(t-t_{0}\right)$ without any significant loss of accuracy; this simplification will be applied in all expressions below involving the sidereal rotation factor $e^{-i \phi}$ or its powers.

From eq. (2) it can be seen that $\vec{e}_{C}=\vec{e}_{Z^{\prime}}$ meaning that the celestial location of the CEP is now that described by the precession/nutation model, 
and is fully predictable. The whole perturbation of the terrestrial pole in space, where perturbation means the difference actual position-model, is expressed in the terrestrial frame as

$$
p=p_{o}+p^{\prime}=p_{o}-P e^{-i \phi},
$$

where $p_{o}=x_{p}-i y_{p}$ is the term considered as polar motion in eq. (1). This is the so-called polar motion gauge in labelling polar motion and nutation, according to the terminology of Eubanks (1993).

Let us compare now the expressions (1) and (2). The entire transformation matrix is the same in both cases and it is expressed by the same 5 variables $x_{p}, y_{p}, X^{\prime}, Y^{\prime}, \mathrm{UT} 1$ - UTC. Note, however, that $X^{\prime}, Y^{\prime}$ have different interpretations in eq. (1) and eq. (2) (see discussion below). Consequently, the partial derivatives of the transformation with respect to all variables are the same. Therefore there is no need to change either the estimation procedure or the observation strategy when switching from the first expression to the second one. The fundamental difference between the transformations (1) and (2) is that they employ different intermediate poles of reference, and that in the second case there is no more ambiguity in the definition of the CEP. This is a strong argument in favour of eq. (2) as an expression of the coordinate transformation between the celestial and the terrestrial reference frames, and for defining the CEP. This approach will be applied throughout the rest of the paper.

If we assume that both $p_{o}$ and $P$ are slowly varying quantities, which is in the spirit of the CEP definition given by Seidelmann (1982), then $p^{\prime}$ in eq. (5) expresses nearly diurnal retrograde variations in polar motion. (The phrase "slowly varying" in this sentence and also whenever used in this paper, means with periods longer than 2 days or with frequencies between -0.5 and 0.5 cpsd (cycles per sidereal day), which should be clear from the rest of this paragraph). If $p_{o}$ and $P$ are estimated simultaneously (this is the case of the routine VLBI determinations) with sampling interval $\Delta$ days (sidereal), then the frequency ranges covered by $p_{o}$ and $p^{\prime}$ are the Nyquist intervals $\Omega(-1 / 2 \Delta, 1 / 2 \Delta)$ and $\Omega(-1-1 / 2 \Delta,-1+1 / 2 \Delta)$, respectively. Maximum frequency coverage by $p$ is obtained for $\Delta=1$, equal to $\Omega(-1.5,0.5)$. For subdiurnal sampling the frequency ranges for $p_{\circ}$ and $p^{\prime}$ become overlapping. Therefore the model described by eq. (5) is no longer directly applicable in this case. An extension of this model for higher frequencies will be discussed in Section 4.

If we consider eq. (4) from the point of view of time series analysis, then $-P$ can be interpreted as the so-called complex demodulate of polar motion at diurnal retrograde frequency $-\Omega$.

\subsection{CEP versus instantaneous rotation pole}

The instantaneous rotation vector can be expressed in the TRS using the perturbation form introduced by Munk and MacDonald (1960)

$$
\vec{\omega}=\Omega\left(m_{1}, m_{2}, 1+m_{3}\right)^{T} .
$$

The corresponding pole, the instantaneous pole of rotation (IPR), described by the complex parameter $m=m_{1}+i m_{2}$, can be expressed in terms of the reported 
quantities, the Earth orientation parameters, using the following relationship derived by Brzeziński (1992)

$$
m=p-i \frac{\dot{p}}{\Omega}
$$

see also (Brzeziński and Capitaine, 1993) for extensive discussion.

If we split up $m$ in the same way as $p$ :

$$
m=m_{0}-m^{\prime} e^{-i \phi},
$$

then eq. (7) applied separately to $p$ and $p^{\prime}$ yields

$$
m_{o}=p_{o}-i \frac{\dot{p}_{o}}{\Omega}, \quad m^{\prime}=-i \frac{\dot{P}}{\Omega} .
$$

Eq.(7) expresses the well-known fact that the IPR is not directly observed by the space geodetic techniques (also by the optical astrometry), therefore using it as a reference pole involves differentiation of the empirical data which is always an undesirable operation amplifying the high frequency noise.

\section{Extension for high frequency perturbations}

Parameterization of the CEP expressed by eq.(5) is no longer representative because it leaves out of consideration important components of polar motion such, as diurnal prograde and semidiurnal variations having both physical explanation and observational evidence. One possibility is to suppress the second term expressing nearly diurnal retrograde variations, which would enable subdiurnal sampling of polar motion. Another possibility, proposed recently by Mathews (1998), is to extend model (5) by adding a separate term expressing diurnal prograde variations, and another one for semidiurnal prograde variations, etc. We will follow this idea below with special emphasis on the consequences for geophysical interpretation of the modern polar motion data.

\subsection{New parameterization of the CEP}

Let us consider the following model for polar motion of the CEP corresponding to the proposal of Mathews (1998)

$$
p(t)=\sum_{n=-N-1}^{N} p_{n}(t) e^{i n \phi},
$$

in which the complex parameters $p_{n}(t)=x_{p, n}(t)-i y_{p, n}(t)$ are assumed to be slowly varying quantities with the cut-off frequency $\Omega / 2$, and $\phi=\phi_{0}+\Omega\left(t-t_{0}\right)$. In terms of the real-valued parameters this model reads

$$
\begin{aligned}
& x_{p}=\sum_{n=-N-1}^{N}\left(x_{p, n} \cos n \phi+y_{p, n} \sin n \phi\right), \\
& y_{p}=\sum_{n=-N-1}^{N}\left(-x_{p, n} \sin n \phi+y_{p, n} \cos n \phi\right) .
\end{aligned}
$$


Note that for $N=0$ this model reduces to the decomposition (5) considered so far with $-P$ replaced by $p_{-1}$.

The $n$th term of expansion (10) describes harmonic oscillation with frequency $n \Omega$ (low frequency for $n=0$, diurnal prograde/retrograde for $n=1 /-1$, semidiurnal prograde/retrograde for $n=2 /-2$, etc.), which subject slow modulation expressed by the time-dependent amplitude $p_{n}(t)$. The frequency range covered by the $n$th term is $\Omega(n-1 / 2, n+1 / 2)$. There is no overlapping between different terms and the total frequency range corresponding to expansion (10) is $\Omega(-N-3 / 2, N+1 / 2)$.

By suppressing $x_{p}^{\prime}, y_{p}^{\prime}$ in eq. (2) and inserting for $x_{p}, y_{p}$ eq. (11), we obtain the coordinate transformation expressed in terms of the new variables. This transformation, together with the $a$ priori models for the motion of the CEP relative to the CRS and to the TRS, can be used to estimate $x_{p, n}(t), y_{p, n}(t)$ directly from the space geodetic measurements. Of course, before doing that it is necessary to modify the data analysis algorithms. Such an attempt using VLBI observations was reported by Bizouard et al. (1999). Note that for some monitoring techniques in which the diurnal retrograde variations in polar motion are fully correlated with perturbations of the orbital elements (GPS, Satellite Lase Ranging - SLR), it may be appropriate to suppress the term $n=-1$ from the model (10)-(11), and add to the $a$ priori model the corresponding empirical corrections determined by VLBI.

But even if the transformation (2) is applied only with the 3 classical parameters $x_{p}, y_{p}, \mathrm{UT} 1-\mathrm{UTC}$, that means after removing diurnal retrograde terms $x_{p}^{\prime}, y_{p}^{\prime}$, and with the subdiurnal resolution, the model $(10)-(11)$ can be accomplished numerically. The parameters of the $n$th component are estimated by the so-called complex demodulation procedure which consists of multiplying the input time series $p(t)=x(t)-i y(t)$ by the factor $e^{-i n \phi}$ and appropriate smoothing. Note however, that for resolving the high frequency terms it is necessary that the sampling interval of the series is less than half of the shortest period in the model.

We chose asymmetrical summation limits in the extended model (10) in order to have symmetry with respect to the initial model (5). In some cases, however, such as when the model is fitted to the subdiurnal time series (this is always the case for the excitation function - see Sec. 4.3), it may be more natural and more convenient to perform the summation from $-N$ to $N$. The value of $N$ depends on the quality of measurements and on the assumed accuracy of the model. At present, the choice $N=2$ seems to be reasonable because the model includes all diurnal/semidiurnal variations with respect both to the TRS and to the CRS. This yields 6 complex parameters $\left(p_{n}\right)$ or, equivalently, 12 real parameters $\left(x_{p, n}, y_{p, n}\right)$ for polar motion, which can be estimated as daily averages. Note that UT1 may also be subjected to a similar parameterization in order to account for diurnal and subdiurnal perturbations, which would further increase the number of the Earth orientation parameters.

Decomposition (10) is based on the implicit assumption that there is no significant power in polar motion around the critical frequencies $\Omega(1 / 2+n), n=$ $0, \pm 1, \pm 2, \ldots$, which are the common edges of the subsequent frequency intervals. This assumption is reasonable on the basis of the current knowledge about polar motion. In particular: 1) all of the known Earth's rotational eigenfrequencies 
are either near $\sigma=0(\mathrm{CW}, \mathrm{ICW})$ or near $\sigma=-\Omega(\mathrm{FCN}, \mathrm{FICN}) ; 2)$ seasonal excitations are low frequency effects; 3 ) all variations of tidal origin are grouped around the frequencies $\sigma=n \Omega$ with integer $n$, which are central frequencies of the terms of expansion $(10) ; 4)$ the only atmospheric normal modes having detectable influence on polar motion are the Rossby-Haurwitz waves $\psi_{3}^{1}, \psi_{1}^{1}$ with frequencies of about $-0.1 \Omega,-0.8 \Omega$, respectively (Brzeziński and Petrov, 1999).

\subsection{Relationship between the CEP and the IRP}

Let us decompose polar motion of the instantaneous rotation axis in a manner similar to what has been done for the CEP in eq. (10)

$$
m(t)=\sum_{n=-N-1}^{N} m_{n}(t) e^{i n \phi},
$$

where the complex amplitudes $m_{n}(t)$ satisfy similar assumptions as $p_{n}(t)$. That means these are slowly varying quantities with the cut-off frequency $\Omega / 2$, and $\phi=\phi_{o}+\Omega\left(t-t_{o}\right)$.

From the assumptions concerning decompositions (10) of $p$ and (12) of $m$ it is rather obvious that the relationship (7) can be applied separately to the corresponding terms of the expansion. After substitution and some simple algebra, the rotation factor $e^{i n \phi}$ on both sides cancels out and we are left with the following first-order relationship

$$
m_{n}=p_{n}(1+n)-i \frac{\dot{p}_{n}}{\Omega},
$$

which for $n=0,-1$ reduces to eq. (9) with $-P,-m^{\prime}$ replaced by $p_{-1}, m_{-1}$, respectively, as would be expected.

\subsection{Dynamics of the CEP}

The conservation of angular momentum yields the equation of polar motion, which can be written in the following general form

$$
\mathcal{F}(m, \chi)=0,
$$

where $\mathcal{F}$ is an abbreviation of the linear differential operator, $m=m_{1}+i m_{2}$ describes polar motion of the instantaneous rotation axis, as defined by eq. (6), and $\chi=\chi_{1}+i \chi_{2}$ is the equatorial excitation function. After substituting eq. (7) for $m$, eq. (14) can be expressed as the ordinary differential equation involving the reported quantities, the terrestrial coordinates of the CEP

$$
\sum_{l=0}^{r} a_{l} p^{(l)}=\sum_{j=0}^{s} b_{j} \chi^{(j)}, \quad \text { with } \quad a_{r} \neq 0, \quad b_{s} \neq 0,
$$

in which the superscript $(k)$ stands for the $k$ th time derivative and $a_{l}, b_{j}$ are constant coefficients. 
A natural further step is to split up the excitation function in the same way as it has been done for $p$ and $m$

$$
\chi(t)=\sum_{n=-N-1}^{N} \chi_{n}(t) e^{i n \phi} .
$$

Equation (15) is linear and, therefore, can be applied separately to the corresponding terms of the expansions (10) and (16). Before doing that, we will perform standard factorization of eq. (15) which yields

$$
\left(\partial_{t}-i \sigma_{1}\right) \cdots\left(\partial_{t}-i \sigma_{r}\right) p=b\left(\partial_{t}-i \omega_{1}\right) \cdots\left(\partial_{t}-i \omega_{s}\right) \chi
$$

where $\partial_{t}$ denotes the time derivative operator, $b=b_{s} / a_{r}, \sigma_{l}$ are the angular eigenfrequencies which are resonant frequencies for the excitation of polar motion. The frequencies $\omega_{j}$ play a similar role when solving the inverse problem, inferring $\chi$ from the observed $p$.

After substituting in eq. (17) the $n$th term of (10) and (16), we derive

$$
\left(\partial_{t}-i \sigma_{1, n}\right) \cdots\left(\partial_{t}-i \sigma_{r, n}\right) p_{n}=b\left(\partial_{t}-i \omega_{1, n}\right) \cdots\left(\partial_{t}-i \omega_{s, n}\right) \chi_{n},
$$

where $\quad \sigma_{l, n}=\sigma_{l}-n \Omega$ and $\omega_{j, n}=\omega_{j}-n \Omega$, and the rotation factor $e^{i n \phi}$ appearing on both sides of the equation has been cancelled out.

Comparison with eq. (17) shows that the only difference is that all characteristic frequencies have been changed by subtracting $n \Omega$. That is now they are referred to the central frequency of the $n$th term. Of course, the same rule of transformation applies to the corresponding frequency domain equation.

Eq. (18) relates slowly varying functions $p_{n}$ and $\chi_{n}$ representing for $n \neq$ 0 high frequency variations in polar motion and in the equatorial excitation function (nearly diurnal prograde/retrograde for $n=1 /-1$, nearly semidiurnal prograde/retrograde for $n=2 /-2$, etc.). This gives us the advantage that these variations can be investigated numerically using much smaller data sets than when using the standard approach because, according to the assumptions, it is sufficient to sample $p_{n}, \chi_{n}$ with the interval of 1 day (or even longer in some cases). We applied such a method to study atmospheric effects on nutation over the data span of about two decades (Bizouard et al. 1998).

Another advantage of the present approach is that the response of the Earth to the applied excitations is different at various frequency regions, therefore different simplifications of eq. (18) are allowed depending on $n$. When considering the low frequency component $(n=0), e . g$, the liquid core effects expressed by the FCN resonance can legitimately be neglected, as it is done in the well-known polar motion equation of Munk and MacDonald (1960). On the other hand, in the analysis of diurnal retrograde variations the FCN plays a very important role and should be taken into account, while the influence of the Chandler resonance can be significantly simplified; see (Brzeziński, 1994) for extensive discussion.

Finally, let us note that we used here a general form of the polar motion equation, eq. (15), bearing in mind that the presently used equations may be still refined in the future in order to fulfill the increasing accuracy requirements. A particular case of the broadband Liouville equation is described with details, including transformation to the diurnal retrograde band and various simplifications, by Brzeziński (1994). An application of this equation to the six-hourly 
time series of the atmospheric angular momentum data in order to estimate the influence upon the nutation amplitudes, was reported by Bizouard et al. (1999).

\section{Concluding remarks}

Our preferred concept for the definition of the Celestial Ephemeris Pole is based on the assumption that its motion in the celestial frame, nutation, comprises only predictable variations due to the gravitational interactions of the Earth with the Moon, the Sun and the planets, while other perturbations caused by the internal processes within the system Earth+ocean+atmosphere, are considered as the terrestrial motion, that is polar motion. This definition does not contain any ambiguity and, according to equations (2) to (4) and following comments, all the historical EOP time series can be easily converted to the new convention without any computational effort.

We considered in details the extended realization of the CEP which takes into account the high frequency perturbations. The underlying model of polar motion, eq. (10), consists of a sum of sinusoidal oscillations with frequencies $n \Omega$ which are multiples of the diurnal sidereal frequency, and multiplied by the time-dependent amplitudes. These amplitudes, the new EOP, are slowly varying in time and can be estimated as daily averages from the intensive measurements by space geodesy. The idea of the model is that instead of shortening the sampling interval for the polar motion coordinates in order to express the high frequency variations, we keep the sampling interval of 1 day or longer but introduce additional parameters ( 2 for each term) expressing nearly diurnal prograde/retrograde oscillations, nearly semidiurnal prograde/retrograde oscillations, etc. motion.

We give several arguments in favor of this new approach to modeling polar

- All the important features of polar motion and its excitation function appear with frequencies in the vicinity of the frequencies $n \Omega$ with integer $n$. Therefore, in this model they are mapped on low frequency variations of the parameters.

- Such a model can easily be accomplished numerically for the subdiurnal estimates of polar motion or of the excitation function. The method, the so-called complex demodulation procedure, consists of multiplying the input time series by the factor $e^{-i \phi}$ and smoothing appropriately.

- This model is also convenient for geophysical interpretation of the observed rotational perturbations. We demonstrated that the linear differential equation of polar motion can also be decomposed into the series of equations eq. (18) which are applicable to the individual terms of the expansion. Each of the equations relates slowly varying variables, which reduces the number of data used in computations in comparison to the standard approach. These equations can also be usually simplified taking into account variability of the transfer function at the corresponding frequencies. 
Acknowledgments. This research has been supported by the Polish National Committee for Scientific Research (KBN) under grant No. 9 T12E 01315.

\section{References}

Bizouard Ch., Brzeziński A., \& Petrov S. D. 1998, Journal of Geodesy, 72, 561. Bizouard Ch., Gontier A.-M, \& Mathews P. M. 1999, presented at XXIth IUGG General Assembly, Birmingham.

Brzeziński A. 1992, manuscripta geodaetica, 17, 3.

Brzeziński A. 1994, manuscripta geodaetica, 19, 157.

Brzeziński A. \& Capitaine N. 1993, Journal of Geophys. Res., 98, No. B4, 6667.

Brzeziński A. \& Capitaine N. 1995, in Proc. Journees Systemes de Reference Spatio-Temporels 1995, Space Res. Centre PAS, Warsaw, 53.

Brzeziński A. \& Petrov S. D. 1999, IERS Technical Note, submitted.

Capitaine N. 1986, ABBA, 162, 323.

Capitaine N. \& Brzeziński A. 1998, in Proc. Journées Systèmes de Référence Spatio-Temporels 1998, Paris Observatory, 155.

Capitaine N., Williams J. G., \& Seidelmann P. K. 1985, A\&A, 146, 381.

Eubanks T. M. 1993, in Contributions of Space Geodesy to Geodynamics: Earth Dynamics, Geodynamics Series, Vol. 24, D. E. Smith \& D. L. Turcotte, American Geophysical Union, Washington, D.C., 1.

Mathews P. M. 1998, in Proc. Journées Systèmes de Référence Spatio-Temporels 1998, Paris Observatory, 161.

McCarthy D. D. (ed.) 1996, IERS Technical Note 21, Paris Observatory.

Munk W. H. \& MacDonald G. J. F. 1960, The Rotation of the Earth: A Geophysical Discussion, Cambridge Univ. Press (reprint 1975).

Seidelmann P. K. 1982, Cel. Mech., 27, 79. 